\title{
Individuelle (Sprach)Lernberatung - eine Hilfe zur Selbsthilfe für «autonome» Lerner in spe
}

TIWA CLABBSEN

Universidad Pablo de Olavide, Sevilla/DAAD

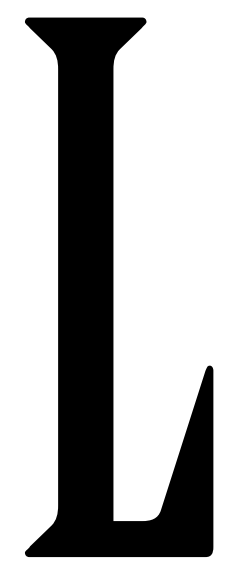

ebenslanges Lernen, Lernerautonomie, selbstgesteuertes oder selbstverantwortliches Lernen sind Schlagworte, ohne die keine bildungspolitische Forderungen oder neuen Curricula mehr auskommen. Ein aktuelles Beispiel ist die Umbenennung des SokratesProgramms in Lebenslanges Lernen ( $L L L)$ im vergangenen Jahr. Gerade im Bereich des Fremdsprachenlernens kommt der Fähigkeit des selbstgesteuerten Lernens eine zentrale Rolle zu, da Sprachen zum einen selbst Veränderungen unterliegen, welche sich der Lerner in der Regel selbstständig erarbeiten muss, und zum anderen das Lernen einer Fremdsprache einen großen Arbeitsaufwand erfordert, der zu einem großen Teil außerunterrichtlich erfolgen muss. Hinzu kommen auch Veränderungen in den Lehrplänen, so auch in den spanischen Universitäten, in denen nach der Einführung der neuen Studienpläne für grado und posgrado bzw. master der Präsenzunterricht zu Gunsten von Selbstlernanteilen gekürzt wird. Nicht alle Lerner sind in der Lage, ihren Lernprozess eigenverantwortlich zu organisieren und brauchen in der Regel Hilfestellung. Eine Möglichkeit die Fähigkeit des selbstgesteuerten Lernens zu fördern stellt die individuelle Lernberatung dar, in deren Theorie und Praxis der folgende Beitrag einführen will.

\section{Beratungskonzept}

Ich stütze mich im Folgenden auf das Konzept einer nicht-direktiven, Autonomie för-

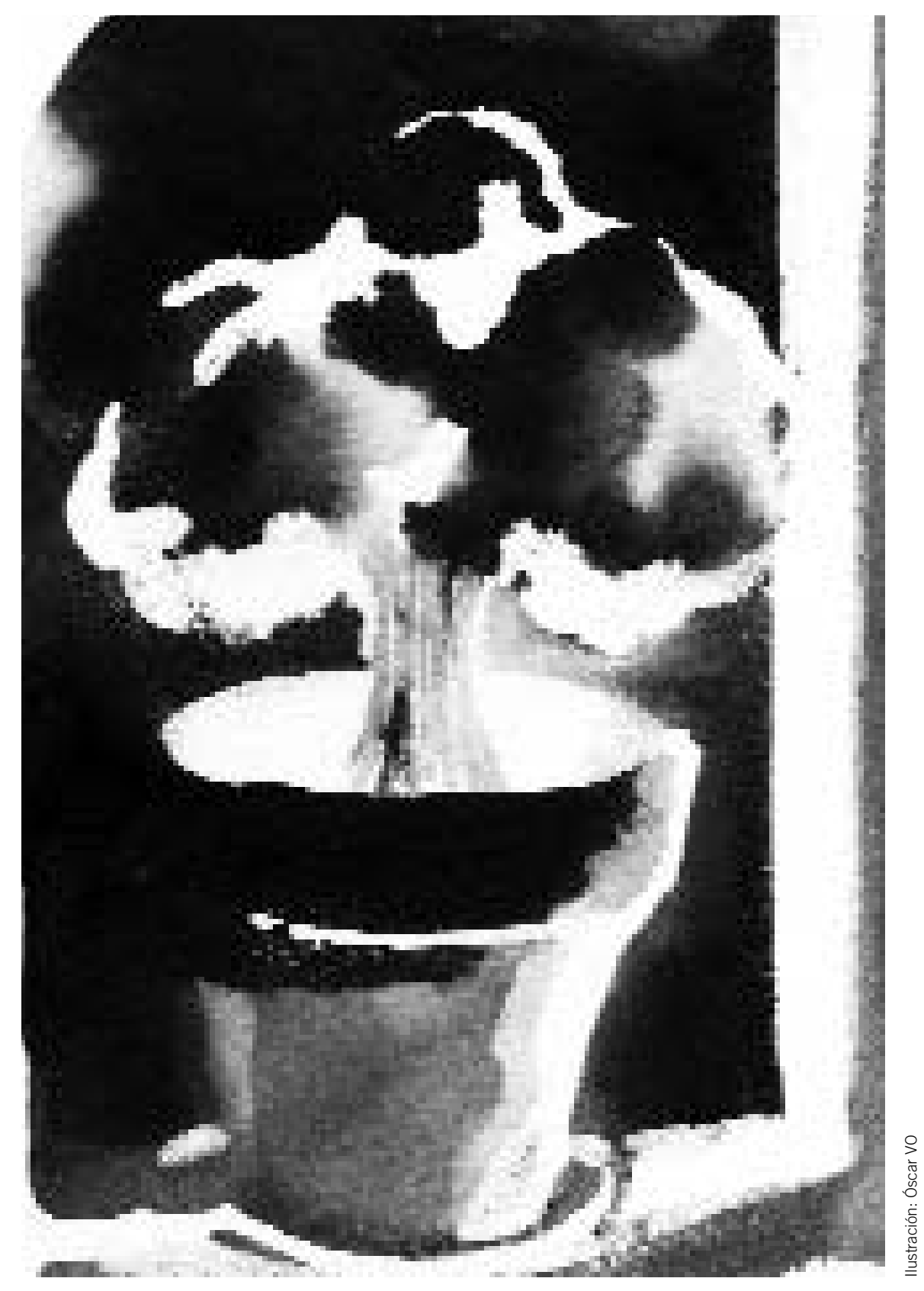




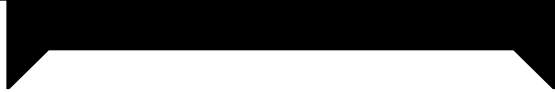

Individuell heißst: “Dem Lerner steht eine ande-

re Person -in der Regel ein Lernexperte—zur

Verfiugung, deren Aufgabe es ist, sich aufi hn als

Personeinzulassenundihmmdabeizuhelfen,selbst-

verantwortlichEntscheidungen so zutreffen,daß

sie sich an seinen individuellen Voraussetzun-

gen und Beduirfnissen ableiten lassen, und ihn

dernden, individuellen Sprachlernberatung, die ein Mittel zur Unterstützung Lernender in konkreten Lernprozessen ist (vgl. exemplarisch Bachmair et al. 1999; Brammerts, Calvert und Kleppin 2001; ClauBen und Peuschel 2006; Kelly 1996; Gremmo 1995; Kleppin 2001, 2003, 2004; Riley 1997; Rogers 1985).

Nicht-direktiv heißt, «...dass das Gespräch zwischen Beraterin und Studierendem möglichst nicht hierarchisch, sondern auf einer Ebene verlaufen soll und die Beraterin den Beratungsprozess nicht zu sehr steuert» (Mehlhorn et al 2005: 161).

Individuell heißt: «Dem Lerner steht eine andere Person -in der Regel ein Lernexperte- zur Verfügung, deren Aufgabe es ist, sich auf ihn als Person einzulassen und ihm dabei zu helfen, selbstverantwortlich Entscheidungen so zu treffen, daß sie sich an seinen individuellen Voraussetzungen und Bedürfnissen ableiten lassen, und ihn dann in seinen Entscheidungen zu stärken» (Brammerts, Calvert und Kleppin 2001: 53).

Autonomie fördernd heißt, selbstverantwortliches und selbstbewusstes Lernen im weiteren wie im engeren Sinne zu fördern Bezug nehmend auf den Autonomiebegriff von Holec bedeutet das, Verantwortung für sein eigenes Lernen zu übernehmen (vgl. Schmelter 2004:264). Dazu gehört die Fähigkeit, Entscheidungen über Lernziele, wege und -inhalte zu treffen und den Lernerfolg anhand der eigenen Bedürfnisse evaluieren zu können.

\section{Ziele und Funktionen der individuellen Lernberatung}

Dem Konzept der individuellen Lernberatung inhärent ist das übergeordnete Ziel, Lerner zum selbstgesteuerten Lernen zu

\section{dann in seinen Entscheidungen zu stärken»}

(Brammerts, Calvert und Kleppin 2001:53).

ermutigen, zu befähigen sowie ihn im selbstgesteuertem Lernprozess zu unterstützen (Brammerts/Calvert/Kleppin 2001, 54). Beratung soll dem Lerner helfen.

- über die eigenen Lernprozesse mit dem Ziel zu reflektieren, sie selbst steuern zu lernen,

- über die eigenen Lernziele selbst zu entscheiden und diese Entscheidungen kontinuierlich (in Abhängigkeit von veränderten Voraussetzungen und Bedürfnissen) zu überprüfen.

- für sich sinnvolle Lernmöglichkeiten (...) zu finden und optimal auszunutzen und die eigenen Lernfortschritte einzuschätzen. (Brammerts, Calvert, Kleppin 2001: 54).

Nach Brammerts, Calvert und Kleppin (2001: 55) muss sich der Lerner beraten lassen,

- weil er zwar prinzipiell Zugang zu allen seinen persönlichen Entscheidungsbedingungen (Ziele, Erfahrungen, Gewohnheiten, Möglichkeiten, Vorlieben usw.) hat, sie aber kaum alle gleichzeitig sehen kann;

- weil er allein kaum alle relevanten $\mathrm{Zu}$ sammenhänge zwischen diesen Bedingungen überblicken kann.

- weil es ihm vielleicht nicht leicht fällt, eigene Entscheidungen in die Tat umzusetzen.
Die ersten beiden Gründe beziehen sich auf eine unzulängliche Fähigkeit, die eigene Bedürfnislage zu diagnostizieren, während der dritte Grund stärker Persönlichkeitsmerkmale in den Blick nimmt (vgl. Schmelter 2004: 326). Lerner, die sich in selbstgesteuerten Lernprozessen befinden, sind sich oftmals nicht sicher, ob die von ihnen getroffenen Entscheidungen richtig sind und/oder wollen diesen durch ein Festlegen unter Zeugen (hier der Berater) einen eher verpflichtenden Charakter geben (Brammerts, Calvert und Kleppin 2001: 60).

Im alläglichen Wortverständnis wird Beratung im Deutschen häufig mit schwer zulösenden Problemen assoziiert-gebräuchlich sind Begriffe wie Steuerberatung. Drogenberatung, Rechtsberatung etc. Das Lösen von Problemen ist aber nur eine Funktion der hier beschriebenen Lernberatung. Sie richtet sich, wie in den obigen Ausführungen deutlich wurde, nicht ausschließlich an Lerner mit erheblichen Lernschwierigkeiten und versteht sich nicht in erster Linie als Problemlösungsinstanz. Vielmehr macht Lernberatung die Lerner mit neuen Lerntechniken vertraut, ermuntert da$\mathrm{zu}$, sie auszuprobieren und für sich zu bewerten; sie dient als Ideenpool und Horizonterweiterung. Nicht zuletzt ist eine weitere zentrale Funktion der Lernberatung das Aufzeigen von Lernfortschritten des Lerners - eine wichtige Voraussetzung für die Aufrechterhaltung der Motivation.

\section{Rolle des Lehrers und Rolle des Beraters}

Betrachtet man die Ziele und Funktionen von Lernberatung sowie das hier skizzierte Beratungskonzept genauer, zeigen sich deutliche Unterschiede in der individuel- 
len Ausgestaltung der Beraterrolle und der Lehrerrolle. Zwar sollten auch Lehrende, die sich eher einem lernerzentrierten Unterrichtskonzept zuordnen würden, über die genannten Beratereigenschaften verfügen und sich auch in ihrem Sprachunterricht an den genannten Prinzipien der Beratung orientieren, ein Unterschied besteht aber darin, dass die Rahmenbedingungen der Institution (Prüfungsvorbereitungen etc.) und die Lernergruppen (z.B. Anzahl der Teilnehmer) ein Einlassen auf den individuellen Lerner erschweren. Außerdem kann ein Unterricht auch von einem Lehrer erfolgreich durchgeführt werden, der sich nicht an den beschriebenen Prinzipien orientiert und der nicht über die Eigenschaften eines nicht-direktiven Beraters verfügt.

Ein lernerorientierter Unterricht hingegen geht mit einer veränderten Lehrerrolle einher. Die Lehrkraft muss ihre Rolle zwischen den «Polen 'Fremdsprachenlehrer' und 'Betreuer selbständigen Lernens'» definieren (vgl. Riley 1997). Als Fremdprachenlehrer hat er nicht nur die volle Verantwortung für seinen Unterricht und dessen Erfolg, er hat auch die gesamte Arbeit der Planung, Vorbereitung und Durchführung. Er ist derjenige, der die Ergebnisse der Lerner kontrollieren und bewerten muss. Als Betreuer selbständigen Lernens bzw. als Berater wird dem Lerner die Verantwortung für seinen Lernprozess überlassen bzw. da viele Lerner nicht in der Lage sind, die Verantwortung für ihr Lernen zu übernehmen, ist eine wichtige Aufgabe des Beraters, sie dabei zu unterstützen und sie schrittweise dazu zu befähigen.

\begin{tabular}{|c|c|}
\hline Berater & Lehrer \\
\hline $\begin{array}{l}\text { Beraterziele } \\
\text { - hilft dem Lerner, Verantwortung für das eigene Lernen } \\
\text { zu übernehmen und beim Lernen autonomer zu werden }\end{array}$ & $\begin{array}{l}\text { Lehrerziele } \\
\text { - unterrichtet den Lerner, übernimmt } \\
\text { Verantwortung für dessen Lernen }\end{array}$ \\
\hline $\begin{array}{l}\text { Lernziele } \\
\text { - elizitiert die individuellen Lernziele des Lerners } \\
\text { - versucht die Lernziele zu verstehen und } \\
\text { hilft, realistische Wege zu finden } \\
\text { - versucht die Groblernziele des Lerners zu verstehen und hilft } \\
\text { ihm dabei, realistische Feinlernziele und Wege zum Erreichen } \\
\text { des Ziels zu finden }\end{array}$ & $\begin{array}{l}\text { Lernziele / Lehrziele } \\
\text { - setzt die Feinlernziele, oft auch die Groblernziele, für den Lerner fest } \\
\text { - gebunden an institutionelle Rahmenbedingungen und Curricula }\end{array}$ \\
\hline Lernmethoden & Lernmethoden/Lehrmethoden \\
\hline $\begin{array}{l}\text { - versucht dem Lerner dabei zu helfen, } \\
\text { eigene Lernwege zu finden und zu gehen }\end{array}$ & $\begin{array}{l}\text { - bestimmt Lehr-/Lernmethoden für seinen Unterricht } \\
\text { - entscheidet über Lernmaterialien und } \\
\text { Rahmenbedingungen wie Zeit, Ort und Tempo }\end{array}$ \\
\hline Evaluation des Lernfortschritts & Evaluation des Lernfortschritts \\
\hline $\begin{array}{l}\text { - versucht dem Lerner dabei zu helfen, } \\
\text { seinen eigenen Lernfortschritt zu evaluieren }\end{array}$ & $\begin{array}{l}\text { - überprüft und testet, ob der Lerner die } \\
\text { vorgegebenen Lernziele erreicht hat }\end{array}$ \\
\hline
\end{tabular}

und sich auch die Lerner dieses (Rollen)Wechsels und der Veränderung des Diskurses bewusst sind (Riley 1997). Für Kleppin (2003: 76) ist das Prinzip der Transparenz ein wesentliches Merkmal für die Unterscheidung der beiden Rollen. In der Lehrerrolle wird oftmals nicht dargelegt, warum Unterrichtsthemen und -inhalte auf eine bestimmte Art und Weise unterrichtet werden und warum in unterschiedlichen Situationen verschiedene Ratschläge gegeben werden, was bei der Übernahme der Beraterrolle in jedem Fall erfolgen muss. Somit können durch die Einhaltung der Beratungsprinzipien insbesondere der Transparenz Beratungselemente in den Unterricht integriert werden (vgl. Kleppin 2001).

\section{Ablauf von Beratungen}

Ein lernerorientierter Unterricht hingegen geht mit einer veränderten Lehrerrolle einher. Die Lehrkraft muss ihre Rolle zwischen den "Polen 'Fremdsprachenlehrer' und 'Betreuer selbstän-

digen Lernens'» definieren (vgl. Riley 1997). Als Fremdprachenlehrer hat er nicht nur die volle Verantwortungfïr seinen Unterricht und dessen Erfolg, er hat auch die gesamte Arbeit der Planung, Vorbereitung und Durchfiuhrung.

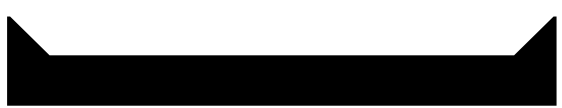

Im Folgenden soll ein prototypischer Ablauf einer individuellen Lernberatung beschrieben werden. Der Beratungsprozess, der in der Regel aus mehreren Beratungsgesprächen bsteht, lässt sich in drei (Makro)Phasen unterteilen. Das einzelne Beratungsgespräch setzt sich wiederum aus drei unterschiedlichen (Mikro)Phasen zusammen.

\section{Makrophasen}

In der ersten Phase des Beratungsprozesses stehen das gegenseitige Kennenlernen und die Schaffung einer vertrauten Gesprächsatmosphäre im Vordergrund. Der Berater erhebt gemeinsam mit dem Lerner dessen Lernerbiographie und stellt die konkreten Lernbedingungen, wie z.B. zu erreichende Ziele, Lernmöglichkeiten, zur Verfügung stehende Zeit usw. zusammen. Wichtig ist in dieser Phase auch die Klärung der Ziele und des Potenzials von Beratung, um den Lerner beispielsweise zu verdeutlichen, dass es sich nicht um eine Nachhilfesituation handelt. In der zweiten Phase des Beratungsprozesses, den eigentlichen Beratungsgesprächen, werden konkrete Bedürfnisse und Ziele des Studierenden besprochen. Diese werden in Verbindung mit den Lernerfaktoren (Lernerbiographie) und den konkreten Lernbedingungen gebracht. Es wird auf bestimmte Verbesserungsmöglichkeiten eingegangen, sehr häufig werden konkrete Lernerprobleme bearbeitet. Die letzte Phase dient der abschließenden Evaluation des Beratungsprozesses. Dabei sollten 
dem Studenten Erfolgserlebnisse und bisher Erreichtes vor Augen geführt und ein positiver Ausblick auf das künftige Vorgehen gegeben werden. Der Lerner ist mittlerweile so unabhängig vom Berater geworden, dass er seine Anliegen allein angehen kann.

\section{Mikrophasen}

Diese drei Phasen können in einer Sitzung mehrfach und in unterschiedlicher Reihenfolge vorkommen (vgl. Brammerts, Calvert \& Kleppin 2001: 57f., Mehlhorn et al. 2005: 167).

Wenn schon ein Lernberatungsgespräch stattgefunden hat, wird zunächst der bisher beschrittene Lernweg gemeinsam evaluiert. Der Lerner berichtet, was er für das Erreichen seiner Lernziele getan hat, wie er mit den verwendeten Lernstrategien - die in der Regel im vorherigen Beratungsgespräch eruiert wurden-zurecht gekommen ist und wie er persönlich seinen Lernerfolg einschätzen würde. Weiterhin muss geklärt werden, welche Lernziele der Lerner erreichen will und/oder für welche (Lern)Probleme er Lösungen sucht. Hierbei stehen die Wünsche, Einschätzungen und Möglichkeiten des Lerners im Vordergrund. Der Berater hilft dem Lerner, sich die eigenen Ziele bewusst zu machen, diffuse oder globale Vorstellungen (zumindest für die nächsten Schritte) zu konkretisieren, widersprüchliche oder unrealistische Einschätzungen zu korrigieren usw. In der nächsten Stufe stehen die Reflexion über die möglichen Wege zu diesen Lernzielen und Problemlösungen im Vordergrund. Auch hier sollte der Lerner selbst zu einer Entscheidung kommen. Aufgabe des Beraters als Experte für das selbstgesteuerte Lernen von Fremdsprachen ist es, dem Lerner durch Einbringen seiner Kenntnisse dabei zu helfen, seine Entscheidungen auf eine solidere Grundlage zu stellen. Am Ende einer Beratungssitzung sollte die Handlungssicherheit des Lerners gestärkt worden sein. Der Berater wird in der Regel insbesondere darauf achten, dass der Lerner klarere Vorstellungen über seine nächsten Lernschritte einschließlich der Verfahren zur Selbstevaluierung entwickelt. An dieser Stelle kann auch eine Vereinbarung zwischen dem Lerner und Berater getroffen werden. Um die Selbstverpflichtung des Lerners zu stei-
Um die Selbstverpflichtung des Lerners zu stei-

gern, ist es sinnvoll, den Lerner am Ende einer

Beratungssitzunguzusammenfassenzulassen,was

er sich zum nächsten Mal vornimmt. Somit wird

auch vermieden, dem Lerner vorschnell Vorge-

hensweisenaufuuoktroyieren, die vonihmselbst

nicht mitgetragen werden können.

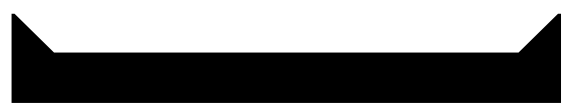

gern, ist es sinnvoll, den Lerner am Ende einer Beratungssitzung zusammenfassen zu lassen, was er sich zum nächsten Mal vornimmt. Somit wird auch vermieden, dem Lerner vorschnell Vorgehensweisen zu oktroyieren, die von ihm selbst nicht mitgetragen werden können.

\section{Formen der Lernberatung}

Lernberatungen können in unterschiedlicher Weise realisiert werden. Hier kann grob zwischen den folgenden Formen unterschieden werden:

- Präsenzberatung vs. Distanzberatung

- Kursunabhängige Beratung vs. kursabhängige Beratung

- Freiwillige Beratungen vs. obligatorische Beratungen

Bei der Präsenzberatung erfolgt die Beratung über ein persönliches Gespräch face to face zwischen Lerner und Berater, während eine Distanzberatung online, über E-Mail oder über das Telefon erfolgt.

Die kursunabhängige Beratung wird dann eingesetzt, wenn eine Beratung ohne Bezug auf einen Sprachkurs zu nehmen angeboten wird oder wenn es sich um einen Lernkontext handelt, der losgelöst von klassischen Unterrichtsformen zu sehen ist, wie z.B. beim Lernen im Individualtandem (im Unterschied zu Tan- demkursen) oder in Selbstlernzentren. Hier ist auch der Beginn und Ursprung von autonomiefördernder Sprachlernberatung zu sehen. So wurde schnell erkannt, dass Lerner allein durch die Schaffung selbstgesteuerter Lernräume und die Bereitstellung von Lernmaterialien, nicht automatisch in die Lage versetzt wurden, eigenverantwortlich und selbstgesteuert zu lernen.

In kursabhängigen Beratungen hingegen werden Inhalte und Fragestellungen aus dem Kurs aufgegriffen, die somit expliziter Beratungsgegenstand sind. Solche Beratungen finden sich in Institutionen wie Kollegs oder Abendschulen, an denen beispielsweise die Studierenden beraten werden, die durch Prüfungen gefallen sind und die die Prüfung im zweiten Anlauf bestehen sollen. Die Beratungen werden dann in der Regel von Lehrkräften durchgeführt, die oftmals auch in den Kursen als Lehrkräfte tätig sind. Dadurch kann es zu Rollenkonflikten kommen, die sich aus den oben dargestellten Unterschieden in der Lehrer- und der Beraterrolle ergeben.

Kursabhängige Beratungen finden sich auch in den Tandemkursen, in denen die Beratung der Tandempaare integraler Bestandteil der Kurse sind (vgl. BlancoHölscher, Kleppin und Santiso Saco 2001). Zu diesen Formen von Beratung lassen sich auch Peerbesprechungen (Helmling 2001, 2006) und die Integrierung von Beratungselementen in den Unterricht zählen (Kleppin 2001).

Ein weiteres Kriterium zur Unterscheidung von Beratungen ist die Freiwilligkeit bzw. Obligatorik. In einigen Universitäten ist die Vergabe eines Leistungsnachweises an den Besuch von Beratungen gekoppelt. So können sich z.B. Studierende an einigen Universitäten in Deutschland und in Spanien ihre Arbeit im Tandem kreditieren lassen, wenn sie innerhalb eines bestimmten Zeitraumes z. B. innerhalb eines Semesters mindestens dreimal einen Berater aufsuchen und ihr Sprachenlernen dokumentieren. An dieser Stelle stellt sich natürlich die Frage, ob der obligatorische Besuch von Beratungen nicht dem Konzept der Lernerautonomie entgegensteht. Meine eigenen Erfahrungen als Beraterin im universitären Kontext und auch die anderer in der Beratungspraxis tätigen Per- 
sonen deuten allerdings darauf hin, dass nur eine Minderheit von Studierenden Beratungsangebote freiwillig aufsuchen und dann hauptsächlich diejenigen, die ihren Lernprozess vergleichsweise gut kontrollieren und reflektieren können. Um hier stichhaltige Auskünfte erteilen zu können, müssen weitere Daten zu den Zielen und Gründen von Lernern eine Beratung aufzusuchen, erhoben werden (Schmelter 2004: 329; Claußen \& Peuschel 2006: 6f.).

\section{Ausblick}

Das in diesem Artikel vorgestellte Konzept der Lernberatung könnte eine effektive Form zur Ausgestaltung der tutorías sein, die mit der Einführung der neuen Studiengänge grado und posgrado auf den Stand einer Unterrichtsform erhoben werden. Vieles deutet darauf hin, dass die Studierenden nach der Kürzung des Präsenzunterrichts professionelle Hilfestellung zur effektiven Nutzung des Selbstlernanteils ihres Studium benötigen werden, gerade dann wenn es nicht nur um die Aneignung bloßen Wissens, sondern -wie

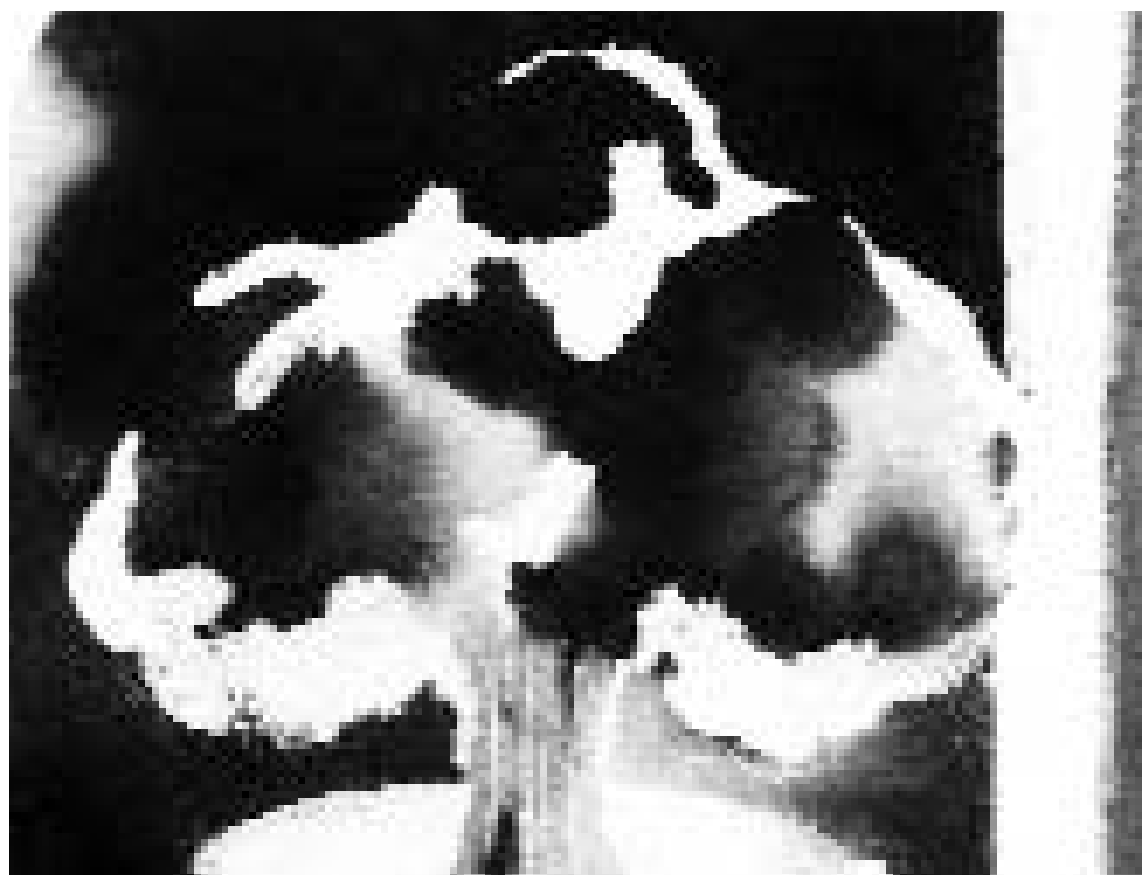

beim Sprachenlernen der Fall- um den Erwerb von Kompetenzen geht.

Aber auch in anderen Sprachlerninstitutionen, die ggf. auch einem Wettbewerb ausgesetzt sind, kann das Angebot einer individuellen Lernberatung ein interessantes Zusatzangebot sein, das dem jeweiligen Kursanbieter anderen Sprachlerninstitutionen gegenüber einen interessanten «Marktvorteil» verschaffen kann

\section{Literatur}

Bachmair, S., Faber, J., Henning, C., Kolb, R. und Willig, W. (1999), Beraten will gelernt sein. Ein praktisches Lehrbuch für Anfänger und Fortgeschrittene. Beltz: Weinheim.

Blanco Hölscher, M., Kleppin, K., Santiso Saco, L. (2001), «Integration von Tandem in fremdsprachliche Curricula: Die Universitäten Bochum und Oviedo", in Brammerts, H. und Kleppin, K. (eds.): Selbstgesteuertes Sprachenlernen im Tandem: Ein Handbuch, Stauffenberg-Verlag, Tübingen, 139-144.

Brammerts, H., Calvert, M., Kleppin, K. (2001), "Ziele und Wege bei der individuellen Lernberatung», in Brammerts, H. und Kleppin, K. (eds.): Selbstgesteuertes Sprachenlernen im Tandem: Ein Handbuch, Stauffenberg-Verlag, Tübingen, 53-60.

Claußen, T. und Peuschel, K. (2006), «Zur Wirksamkeit von individuellen Sprachlernberatun- gen", in Zeitschrift für Interkulturellen Fremdsprachenunterricht [Online], 11 (2), $10 \mathrm{~S}$. Verfügbar unter: http://zif.spz.tu$\mathrm{d}$ a r m s t a d t . d e/jg - 11 2/beitrag/ClaussenPeuschel1.htm

Gremmo, M.-J. (1995), «Conseiller n'est pas enseigner: Le rôle du conseiller dans l'entretien du conseil», in Mélanges CRAPEL, 22, 33-61.

Helmling, B. (2001), «Peergruppenarbeit - Tandems lernen von Tandems». In Brammerts, H. \& Kleppin, K. (eds.) (2001).

Helmling, B. (2006), «Peergruppenarbeit", in Zeitschrift für interkulturellen Fremdsprachenunterricht [Online], 11 (2) 10 S. Verfügbar unter: h t t p: // zif.spz.tu darmstadt.de/jg-112/beitrag/Helmling1.htm.

Kelly, R. (1996), «Language counselling for learner autonomy: the skilled helper in self-access language learning», in Pemberton, R. et al. (eds.) (1996), Taking control: Autonomy in Language
Learning, Hong Kong University Press, Hong Kong, 93-114.

Kleppin, K. (2001), "Vom Lehrer zum Lernberater. Zur Integration von Beratungselementen in den Fremdsprachenunterricht", in Meißner, F-J. und Reinfried, M. (eds.) (2001), Bausteine für einen neokommunikativen Französischunterricht (S. 51-60). Narr, Tübingen, 51-60

Kleppin, K. (2003), «Sprachlernberatung: Zur Notwendigkeit eines eigenständigen Ausbildungsmoduls", in Zeitschrift für Fremdsprachenforschung 14 (1), 71-85.

Kleppin, K. (2004), «Bei dem Lehrer kann man ja nichts lernen". Zur Unterstützung der Motivation durch Sprachlernberatung", in Zeitschrift für interkulturellen Fremdsprachenunterricht [Online], 9(2), 16 $\mathrm{S}$. Verfügbar unter h t t p : // zif.spz.tu darmstadt.de/jg-09. 2/beitrag/Kleppin2.htm.
Holec, H. (1980): Autonomy and Foreign Language Learning. Council of Europe, Strasbourg.

Riley, P. (1997), "The guru and the conjurer: aspects of counselling for self-access", in Benson, P. Voller, P. (eds.) (1997). Autonomy and Independence in Language Learning, Longman, London, 114-131.

Rogers, C. R. (1985), Die nichtdirektive Beratung. Fischer, Frankfurt am Main.

Mehlhorn, G. unter Mitarbeit von Bausch, K.-R., Claußen, T., Helbig-Reuter, B., Kleppin, K. (2005), Studienbegleitung für ausländische Studierende an deutschen Hochschulen. Teil 1: Handreichungen für Kursleiter zum Studierstrategien-Kurs. Teil 2: Individuelle Lernberatung - Ein Leitfaden für die Beratungspraxis, Iudicium, München.

Schmelter, L. (2004), Selbstgesteuertes oder potenziell expansives Fremdsprachenlernen im Tandem, Narr, Tübingen. 


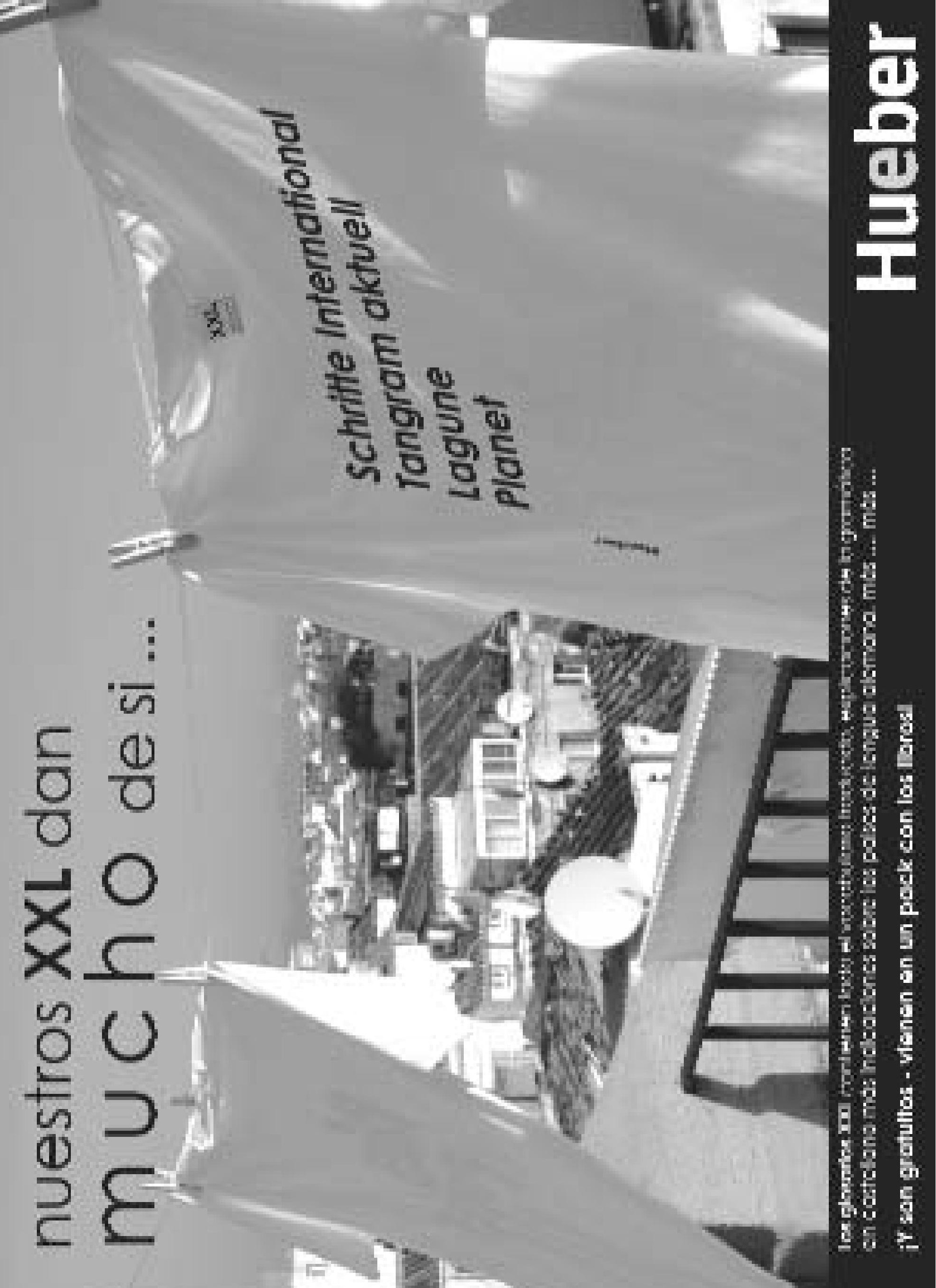

